\title{
Short-term Voltage Stability of Distribution Grids With Medium-scale PV Plants due to Asymmetrical Faults
}

\author{
Monirul Islam ${ }^{1}$, N. Mithulananthan ${ }^{1}$, Jahangir Hossain ${ }^{2}$ and K. Bhumkittipich ${ }^{3}$ \\ ${ }^{1}$ Power and Energy System group, School of ITEE, University of Queensland, Brisbane, Australia \\ ${ }^{2}$ School of Engineering, Macquarie University, NSW-2109, Australia \\ ${ }^{3}$ Department of Electrical Engineering, Rajamangala University of Technology, Pathum Thani 12110, Thailand \\ mdmonirul.islam@uq.edu.au, mithulan@itee.uq.edu.au, jahangir.hossain@mq.edu.au,krischonme.b@en.rmutt.ac.th
}

\begin{abstract}
With the increasing penetration of photo-voltaic (PV) units into electrical grids, particularly in distribution networks (DNs), the concern of short-term voltage instability (STVI) are growing in the presence of induction motor (IM) loads. On the event of unsymmetrical faults, STVI issues could be more complicated as the next-generation $P V$ systems would require negative sequence power injection into the grid in conjunction with positive one. Therefore, this paper comprehensively investigates the impact of negative sequence power on the shortterm voltage stability (STVS) of DNs. The method of characterizing an unbalanced fault and supplementary controls for PV systems are developed. Different case studies are conducted on a balanced IEEE 4 bus and an unbalanced IEEE 13 bus system by injecting different level of negative sequence power considering with and without peak current limitation of the PV converters. It is observed that STVS is likely to be weakened in case of large negative sequence power penetration, while injecting high positive sequence power can cause excessive voltage swell resulting inverter disconnections. Therefore, both positive and negative sequence powers need to be injected optimally to ensure the system's security following a fault.
\end{abstract}

Index Terms-PV inverter, sequence controller, short-term voltage stability, unbalanced faults, and voltage ride-through.

\section{INTRODUCTION}

In recent years, the PV power generation has been increased significantly all around the world, reached total installation of 400GW at the end of 2017 [1]. As most of these inverter-based PV generators are being integrated in DNs, it can raise concerns relating to the reliability and stability of the network, specifically at the event of grid disturbance [2]. Acknowledging this issue, most of the grid codes have been revised including the requirements of fault ride-through and reactive and/or active power injection capability following a grid contingency $[2,3]$. This has been accomplished to support the grid for ensuring the voltage stability both in short and long term periods, while reducing the possibility of a voltage collapse $[2,3]$.

The voltage stability in short-term has been gaining an enormous attention both from academia and industry in recent years due to integration of dynamic loads, for example, induction motor (IM) loads and inverter-based distributed generators (DGs), namely, PV units [4-7]. There are mainly two types of short-term voltage instability (STVI) issues: (1) fault-induced delayed voltage-recovery and (2) voltage instability [5, 8]. It has been reported in many research papers that taking almost 3 to 5 times of rated current during stalling of IM load after a disturbance is the key cause of STVI problems [9]. However, disconnection of PV-inverters which resulted in loss of PV power following a fault could be another reasons of STVI in DNs with large PV penetrations $[6,7]$.

It may be noted that though the power systems, particularly the DNs are being penetrated by a large number of PV generators, very few researches have focused on this issue of how the dynamics of PV systems affect the STVS [4-7]. In [6], it has been concluded that STVI is likely to be occurred owing to loss of PV power or even slower recovery after a fault. Two counter measures i.e. leading power factor operation and reactive power support from PV inverters have been suggested and tested, and a significant improvement of STVS has been observed in case of reactive power support. An interesting observation has been presented in [4] that active power support along with reactive from PV systems would be better solution in STVS augmentation compared to only reactive power injection. In order to thoroughly understand the effects of active power support along with reactive from PV systems on the STVS, a detail study has been accomplished in [5, 7], where the improvement of STVS in DNs with high resistive characteristics as a result of active and reactive power support by PV systems has also been reported. Consequently, several countermeasures based on different control strategies which allow different level of active power penetration into the grid, have been developed in [5, 7] and the provided results evidently designated the enhancement of STVS.

From the earlier discussion, it can be perceived that most of the researches [4-7] have concentrated on the active and reactive power support after a symmetrical fault. However, the probability of arising asymmetrical faults in power systems is higher than the symmetrical one. An unbalance voltage sag is created during asymmetrical faults that may lead to more complicated control. It can be mentioned that though most of the grid codes demand positive sequence power injection, the next generation PV systems might introduce negative sequence power penetration requirement during an asymmetrical faults to reduce voltage unbalance [3]. Therefore, it is necessary to better understand to what extent and under what circumstances the PV systems are required to inject negative sequence power 
and how it's influencing the STVS.

Taking into account the prior issue, in this paper, an intensive investigation is conducted by injecting different level of negative sequence power to thoroughly comprehend its impact on the STVS during an unbalanced fault on the purpose of assisting DN design engineers. First, a method to characterize the asymmetrical faults is presented. Next, the voltage support control strategies through positive and negative sequence power injection are developed followed by the technique of reactive current injection and peak current limitation. Finally, several case studies are conducted without imposing the peak current limit of the inverter on an IEEE 4 bus test system which represents a balance DN. It is observed that the system is secured in terms of STVS as well as highvoltage risk. However, once the peak current limit is applied, the STVI is happened for the cases of high negative sequence current injection. Unlike IEEE 4 bus, presented results from IEEE 13 bus which denotes an unbalanced DN, indicate that the STVI may possibly be occurred in case of high negative sequence current even without clamping the peak current.

The remaining of the paper is structured as follows: the configuration of the grid-tied PV systems and the method to characterize of asymmetrical faults are given in Section II. In Section III, the idea of voltage support and associated controls for unbalanced voltage sag are explained. The details of the case studies, and the results and analysis are presented in Section IV. Section V concludes this research with the highlights of conclusions and contributions.

\section{ASYMMETRICAL FAULTS CHARACTERIZATION}

During an unbalanced fault, the voltage and current at PCC can be characterized as the sum of positive, negative and zero sequence components [10-13]:

$$
\left[\begin{array}{c}
v_{a} \\
v_{b} \\
v_{c}
\end{array}\right]=\left[\begin{array}{c}
v_{a}^{+} \\
v_{b}^{+} \\
v_{c}^{+}
\end{array}\right]+\left[\begin{array}{c}
v_{a}^{-} \\
v_{b}^{-} \\
v_{c}^{-}
\end{array}\right]+\left[\begin{array}{c}
v_{a}^{0} \\
v_{b}^{0} \\
v_{c}^{o}
\end{array}\right]
$$

where the superscripts,+- , and 0 indicate the positive, negative and zero sequence, respectively. In case of a three-phase threewire system, zero sequence components can be neglected as there is no neutral line to ground. After applying Clarke transformation, the PCC voltages are developed in stationary reference-frame (SRF) as follows:

$$
\left[\begin{array}{c}
v_{\alpha} \\
v_{\beta}
\end{array}\right]=\left[\begin{array}{c}
v_{\alpha}^{+} \\
v_{\beta}^{+}
\end{array}\right]+\left[\begin{array}{c}
v_{\alpha}^{-} \\
v_{\beta}^{-}
\end{array}\right]
$$

where $v_{\alpha}^{+}, v_{\beta}{ }^{+}$and $v_{\alpha}^{-}, v_{\beta}^{-}$are the positive and negative sequence voltages at PCC, respectively in SRF. These voltages can be defined as a function of time as:

$$
\left[\begin{array}{c}
v_{\alpha}^{+} \\
v_{\beta}^{+}
\end{array}\right]=V^{+}\left[\begin{array}{c}
\cos \left(\omega t+\varphi^{+}\right) \\
\sin \left(\omega t+\varphi^{+}\right)
\end{array}\right]
$$

$$
\left[\begin{array}{c}
v_{\alpha}^{-} \\
v_{\beta}^{-}
\end{array}\right]=V^{-}\left[\begin{array}{c}
\cos \left(\omega t-\varphi^{-}\right) \\
-\sin \left(\omega t-\varphi^{-}\right)
\end{array}\right]
$$

where $\omega$ symbolizes the grid angular-frequency, $V^{+}, \varphi^{+}$and $V^{-}$, $\varphi^{-}$are the amplitudes and primary phase-angles in terms of positive and negative sequence, respectively. It may be noted that diverse voltage dip as a results of various faults can be characterized by the sequence amplitudes and angles in SRF using the following equations [11]:

$$
\begin{gathered}
V^{+}=\sqrt{\left(v_{\alpha}^{+}\right)^{2}+\left(v_{\beta}^{+}\right)^{2}} \\
V^{-}=\sqrt{\left(v_{\alpha}^{-}\right)^{2}+\left(v_{\beta}^{-}\right)^{2}} \\
\varphi=\varphi^{+}-\varphi^{-}=\cos ^{-1}\left(\frac{v_{\alpha}^{+} v_{\alpha}^{-}+v_{\beta}^{+} v_{\beta}^{-}}{V^{+} V^{-}}\right)
\end{gathered}
$$

The voltage unbalance factor (VUF) that defines the degree of unbalance due to various asymmetrical faults is presented in (8).

$$
u=\frac{V^{-}}{V^{+}}
$$

In the next section, control strategies for PV systems to interact with these unsymmetrical faults have been presented.

\section{VOLTAGE SUPPORT CONTROL}

\section{A. Idea of Voltage Support:}

The mathematical equation of PCC voltage can be presented in (9) in terms of grid voltage and impedance.

$$
v=v_{g}+R_{g} i+L_{g} \frac{d i}{d t}
$$

Considering unbalance scenario, (9) can be separated into two using the symmetrical components of the voltage and current as given in (10) and (11) [3,14].

$$
\begin{aligned}
& V^{+}=V_{g}^{+}+R_{g} I_{p}^{+}+\omega L_{g} I_{q}^{+} \\
& V^{-}=V_{g}^{-}+R_{g} I_{p}^{-}-\omega L_{g} I_{q}^{-}
\end{aligned}
$$

It can be observed from (10) that positive sequence active and reactive power injection would boost the PCC voltage. On the other hand, as seen in (11), voltage unbalance can be mitigated by injecting negative-sequence reactive current, while negative-sequence active current need to be reduced.

\section{B. Voltage Support Control}

According to three-phase power principle, instantaneous active and reactive power can be calculated in (12).

$$
\left[\begin{array}{l}
p \\
q
\end{array}\right]=\frac{3}{2}\left[\begin{array}{cc}
v_{\alpha} & v_{\beta} \\
v_{\beta} & -v_{\alpha}
\end{array}\right]\left[\begin{array}{l}
i_{\alpha} \\
i_{\beta}
\end{array}\right]
$$

In case of unbalanced condition, power components in (12) can be rewritten as: 


$$
\left[\begin{array}{l}
p \\
q
\end{array}\right]=\left[\begin{array}{c}
p^{+} \\
q^{+}
\end{array}\right]+\left[\begin{array}{c}
p^{-} \\
q^{-}
\end{array}\right]+\left[\begin{array}{c}
\tilde{p} \\
\tilde{q}
\end{array}\right]
$$

where $p^{+}, p^{-}$and $q^{+}, q^{-}$are the positive and negative sequence instantaneous active and reactive power, respectively, and $\tilde{p}$ and $\tilde{q}$ are the oscillating terms at twice the grid frequency. The power components in (13) can be calculated in the form of voltage and current in SRF as follows [3]:

$$
\begin{aligned}
& p^{+}=\frac{3}{2}\left(v_{\alpha}^{+} i_{\alpha}^{+}+v_{\beta}^{+} i_{\beta}^{+}\right) \\
& p^{-}=\frac{3}{2}\left(v_{\alpha}^{-} i_{\alpha}^{-}+v_{\beta}^{-} i_{\beta}^{-}\right) \\
& q^{+}=\frac{3}{2}\left(v_{\beta}^{+} i_{\alpha}^{+}-v_{\alpha}^{+} i_{\beta}^{+}\right) \\
& q^{-}=\frac{3}{2}\left(v_{\beta}^{-} i_{\alpha}^{-}-v_{\alpha}^{-} i_{\beta}^{-}\right) \\
& \tilde{p}=\frac{3}{2}\left(v_{\alpha}^{+} i_{\alpha}^{-}+v_{\beta}^{+} i_{\beta}^{-}+v_{\alpha}^{-} i_{\alpha}^{+}+v_{\beta}^{-} i_{\beta}^{+}\right) \\
& \tilde{q}=\frac{3}{2}\left(-v_{\alpha}^{+} i_{\beta}^{-}+v_{\beta}^{+} i_{\alpha}^{-}-v_{\alpha}^{-} i_{\beta}^{+}+v_{\beta}^{-} i_{\alpha}^{+}\right)
\end{aligned}
$$

From (12)-(17), the active and reactive current generator can be formulated as follows $[3,12]$ :

$$
\begin{aligned}
& {\left[\begin{array}{c}
i_{\alpha(p)} \\
i_{\beta(p)}
\end{array}\right]=\frac{2}{3} \frac{P^{+}}{\left(v_{\alpha}^{+}\right)^{2}+\left(v_{\beta}^{+}\right)^{2}}\left[\begin{array}{c}
v_{\alpha}^{+} \\
v_{\beta}^{+}
\end{array}\right]+\frac{2}{3} \frac{P^{-}}{\left(v_{\alpha}^{-}\right)^{2}+\left(v_{\beta}^{-}\right)^{2}}\left[\begin{array}{c}
v_{\alpha}^{-} \\
v_{\beta}^{-}
\end{array}\right]} \\
& {\left[\begin{array}{c}
i_{\alpha(q)} \\
i_{\beta(q)}
\end{array}\right]=\frac{2}{3} \frac{Q^{+}}{\left(v_{\alpha}^{+}\right)^{2}+\left(v_{\beta}^{+}\right)^{2}}\left[\begin{array}{c}
v_{\beta}^{+} \\
-v_{\alpha}^{+}
\end{array}\right]+\frac{2}{3} \frac{Q^{-}}{\left(v_{\alpha}^{-}\right)^{2}+\left(v_{\beta}^{-}\right)^{2}}\left[\begin{array}{c}
v_{\beta}^{-} \\
-v_{\alpha}^{-}
\end{array}\right]}
\end{aligned}
$$

where $P^{+}, P^{-}$and $Q^{+}, Q^{-}$denote the positive and negative sequence active and reactive power reference, respectively.

In order to recognize the flexible power control during fault ride-though (FRT) following unbalanced voltage sag, two control gains such as active $k_{p}$ and reactive $k_{q}$ has been introduced as [15]:

$$
k_{p}=P^{+} / P, \quad k_{q}=Q^{+} / Q
$$

By inserting (3)-(4) and (22) in (20)-(21), the amplitude of the positive and negative sequence current can be calculated from (23)-(26) [3].

$$
\begin{aligned}
& I_{p}^{+}=\frac{2}{3} \frac{k_{p} P}{V^{+}} \\
& I_{p}^{-}=\frac{2}{3} \frac{\left(1-k_{p}\right) P}{V^{-}} \\
& I_{q}^{+}=\frac{2}{3} \frac{k_{q} Q}{V^{+}}
\end{aligned}
$$

$$
I_{q}^{-}=\frac{2}{3} \frac{\left(1-k_{q}\right) Q}{V^{-}}
$$

\section{Reactive Power Reference Calculation}

In this research, for the period of regular operationcondition $\left(0.95<V_{g}<1.05\right)$, assumed that the PV generators penetrate only active power into the grid. However, to meet the grid requirement, the PV inverters inject reactive power into the grid when the system is encountered a fault.

It can be mentioned that different countries have adopted dissimilar reactive power injection requirement in their grid code $[16,17]$. As stated in German grid-code (E.ON), grid-tied inverters are required to inject full reactive power for 0.5 per unit (pu) or more voltage sag condition [16]. The reactive current reference during voltage sag can be calculated in accordance with E.ON grid code as follows:

$$
\left\{\begin{array}{c}
I_{q}=\mu\left(1-V_{g p}\right) I_{n} \quad \text { when } 1-1 / \mu \leq V_{g p} \leq 0.95 \\
I_{q}=I_{n} \text { when } V_{g p}<1-1 / \mu
\end{array}\right.
$$

where $I_{n}$ and $V_{g p}$ represent the nominal grid current and pu instantaneous grid voltage respectively, while $\mu$ defines the volume of reactive current to be injected. In this paper, $\mu$ is selected to be 2 to allow full reactive power injection by PV inverters at $0.5 \mathrm{pu}$ and more voltage dip.

\section{Peak Current Limit Control:}

Considering power semiconductors capabilities, limiting peak current of any grid-tied PV inverters is obligatory to avoid any undesirable trip-off. However, most of the control strategies proposed in the literature for unsymmetrical faults have overlooked this important issue. Though it is very straightforward to design the current limiter for symmetrical faults as no negative-sequence current is present, it becomes more difficult to design the current limiter in case of asymmetrical faults with both positive and negative sequence components.

In order to limit the injected grid current within peak, an indepth analysis has been conducted in [3] by calculating the amplitude of each phase current. It has been shown that three different solutions (one solution at one phase) are available for active power to clamp the grid-current within maximum $I_{\max }$ if the reactive power injection during fault has been given the priority. Therefore, selecting the minimum active power among these three solutions would ensure the phase current within rated value as:

$$
P=\min \left\{P_{a}, P_{b}, P_{c}\right\} \Rightarrow \max \left\{I_{a}, I_{b}, I_{c}\right\}=I_{\text {max }}
$$

Finally, the reactive power solution can be calculated in (29), which has been derived by combining prior three solutions [3].

$$
\begin{aligned}
& P=\frac{-2 x Q+\sqrt{y\left(3 I_{\max } u V^{+}\right)^{2}-(2 z Q)^{2}}}{2 y} \\
& \text { where } \quad x=\left(k_{p}+k_{q}+2 k_{p} k_{q}\right) u \sin (\hat{\varphi})
\end{aligned}
$$




$$
\begin{gathered}
y=k_{q}^{2}\left[1+2 u \cos (\hat{\varphi})+u^{2}\right]-2 k_{q}[1+u \cos (\hat{\varphi})]+1 \\
z=k_{p}[1-u \cos (\hat{\varphi})]+k_{q}[1+u \cos (\hat{\varphi})]+k_{p} k_{q}\left(u^{2}-1\right)-1
\end{gathered}
$$

while $\hat{\varphi}$ is consisting of three different values for obtaining $P_{a}$, $P_{b}$, and $P_{c}$ as:

$$
\hat{\varphi}=\{\varphi, \varphi+2 \pi / 3, \varphi-2 \pi / 3\}
$$

\section{CASe Studies}

In the interest of thoroughly understand the STVS of a highly three-phase PV penetrated DN following asymmetrical faults, several case studies based on sequence reactive power injection are conducted. These case-studies are performed in two IEEE standard test-systems, namely, IEEE 4 bus and IEEE 13 bus test-systems for different scenarios. Case studies are accomplished using Matlab/Simulink software considering unsymmetrical line to line (L-L) fault at substation bus for the period of $0.2 \mathrm{~s}$. All the per-unit parameters are calculated on the basis of $1000 \mathrm{kVA}$ base.

\section{A. Case-studies on IEEE 4 Bus Test-system:}

A modification of the IEEE 4 bus test-system which represents a balanced DN is accomplished by accommodating PV generators and IM loads through a transformer $(\operatorname{Tr} 2)$ as shown in Fig. 1. The pu parameters of the transformers 2 has been selected same as transformers 1 . The specifications of this test-system and the modified loads can be accessible in [18] and Table A1 in Appendix A. The PV units are installed in the network so as aggregate penetration becomes $50 \%$ of total load. The load arrangement is assumed as IM-type $70 \%$ and resistive-type $30 \%$ to reflect a residential network. The pu parameters of the IM loads are tabulated in Table A2 in Appendix A. Five different cases based on reactive control gain that defines the amount of negative-sequence reactive power to be injected, are simulated as shown in Table I.

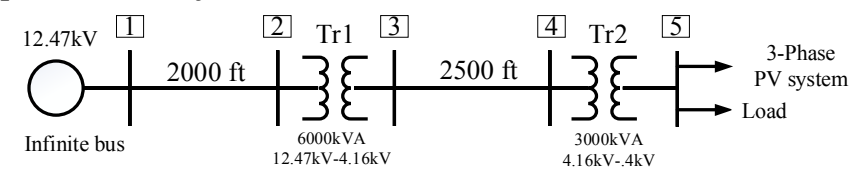

Figure 1. Modified IEEE 4 bus system.

TABLE I. INVESTIGATION CASES

\begin{tabular}{|c|c|c|c|c|}
\hline Cases & $\begin{array}{c}\text { Reactive control } \\
\text { gain } k_{q}\end{array}$ & $\begin{array}{c}\text { Active control } \\
\text { gain } k_{p}\end{array}$ & $\begin{array}{c}\text { PV } \\
\text { Penetration }\end{array}$ & $\mu$ \\
\hline 1 & 0.00 & & & \\
\hline 2 & 0.25 & \multirow{2}{*}{1.0} & $50 \%$ & 2.0 \\
\hline 3 & 0.50 & & & \\
\hline 4 & 0.75 & & & \\
\hline 5 & 1.00 & & & \\
\hline
\end{tabular}

The three-phase dynamic voltage profiles at bus 4 and the output power of PV generators without and with peak current limit are shown in Figs. 2 and 3, respectively. Witnessed by Phase A (top) and C (bottom) voltages in Fig. 2(a), though voltage sag is occurred due to an L-L fault, the system was able to recover the post-contingency voltage to normal operating condition for all cases. Furthermore, increasing voltage recovery speed in Phase $\mathrm{C}$ as well as rising voltage swell in
Phase B is observed for the Cases 5 to 1 respectively as expected. However, the maximum voltage swell is approximately $0.15 \mathrm{pu}$ in Case 5 which is still below the HVRT requirement defines in different grid codes [16, 17]. The scenario of STVS could be different in case of peak current limit conditions. As shown in Fig 2(b), all Phase A, B and $\mathrm{C}$ voltages are likely to be instable in short-term for the Cases 1 to 3 . Nevertheless, voltage recovery to stable condition is ensued in Cases 4 and 5, though delayed recovery is observed in Case 4. This is happened because of the variety of active and reactive power injection which is shown in Fig. 3.
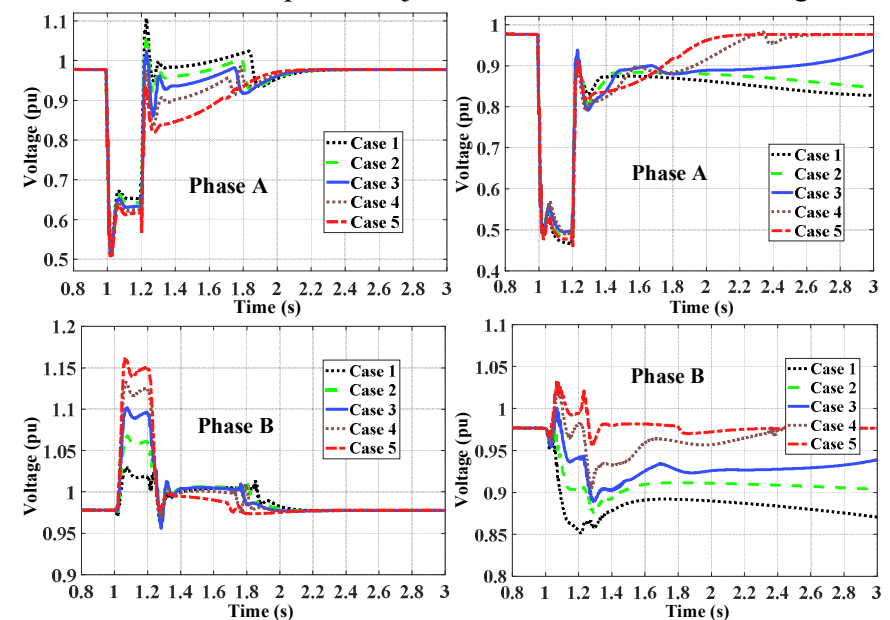

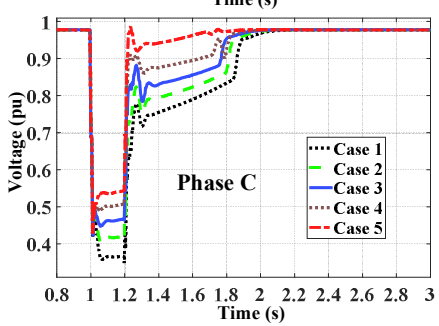

(a)

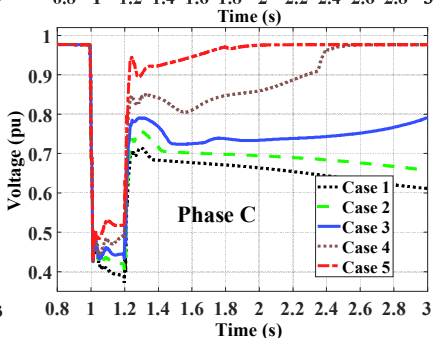

(b)
Figure 2. Voltage dynamics at bus 4: (a) without and (b) with I limit.

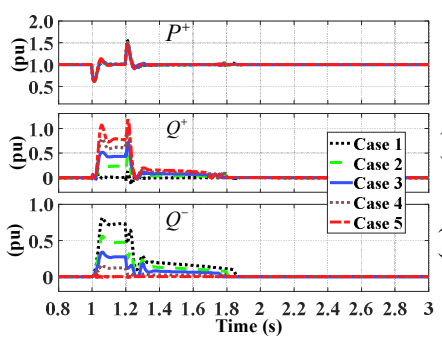

(a)

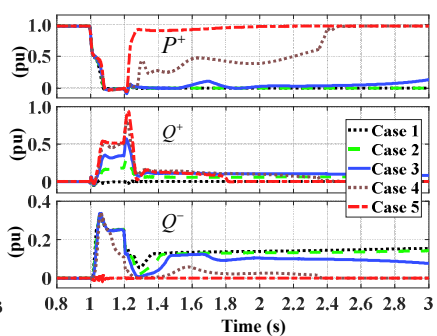

(b)
Figure 3. Positive Sequence active power (top), and positive (middle) and negative (bottom) sequence reactive power : (a) without and (b) with $I$ limit.

It can be perceived from Fig. 3 that without current limit, constant active power was injected in all cases for the entire grid period, while it becomes zero during fault in case of peak current limitation control. According to Fig. 3(b), positive sequence active power has been realized instant recovery subsequent the fault in Case 5, and 1.2s delayed retrieval in Case 4. In contrast, the PV generators was unable to recover the active power in Cases 1 to 3 as to allow reactive power 
injection by keeping peak current within limit, which is the key cause of STVI in Cases 1 to 3 with $I$ limit. Furthermore, different level of positive and negative sequence reactive power injection based on the reactive control gain can be seen in Fig. 3 for both with and without $I$ limit control. It can be revealed that increasing voltage recovery speed in Phase $\mathrm{C}$ and growing voltage swell in Phase B for the Cases 5 to 1 , respectively are happened owing to this diversity of reactive power injection.

Table II shows voltage recovery (ROCOVR), settling time and remarks on stability point of view for different cases when current limit peak has been applied. It may be noted that settling time is the duration from the instant of fault cleared until the voltage reached to $0.95 \mathrm{and} /$ or $1.05 \mathrm{pu}$. ROCOVR is the average of time derivative of the power system voltage following a disturbance during a considered transient time frame, $\mathrm{T}$ which can be calculated based on (34). It should be noted that higher value of ROCOVR means higher recovery speed so as better STVS.

$$
R O C O V R=\frac{T-T_{c}}{\Delta t} \sum_{t=T_{c}}^{T} \frac{d}{d t} V \quad \Delta \mathrm{t} \in\left[T_{c}, T\right]
$$

where $T_{c}$ and $\Delta t$ represent the fault clearing time and step time, respectively. It can be observed that ROCOVR is gradually increased for the Cases 1 to 5 respectively in all phases except Phase B with L-L fault. As the L-L fault occurs between Phases A and C, voltage swell is detected in Phase B which in results decreased ROCOVR in Phase $\mathrm{B}$. On the other hand, settling time is some phases are not available as the voltage was unable to return to $0.95 \mathrm{pu}$, consequently STVI occurs. Furthermore, Phase B (Case 5) has been realized zero settling time which means the voltage persist within 0.95 to $1.05 \mathrm{pu}$.

\begin{tabular}{|c|c|c|c|c|c|c|c|c|}
\hline \multirow{2}{*}{ Fault Types } & \multirow{2}{*}{ Cases } & \multicolumn{3}{|c|}{ ROCOVR } & \multicolumn{3}{|c|}{ Settling time (s) } & \multirow{2}{*}{ Stability } \\
\hline & & $\mathrm{A}$ & $\mathrm{B}$ & $\mathrm{C}$ & $\mathrm{A}$ & B & $\mathrm{C}$ & \\
\hline \multirow{5}{*}{$\begin{array}{l}\text { Single Line to } \\
\text { Ground } \\
\text { (1-L-G) }\end{array}$} & Case 1 & 0.75 & 0.15 & 0.06 & 1.15 & - & - & Unstable \\
\hline & Case 2 & 1.27 & 0.18 & 0.09 & 0.68 & 1.45 & - & Unstable \\
\hline & Case 3 & 1.28 & 0.29 & 0.17 & 0.65 & 0.80 & 1.42 & Stable \\
\hline & Case 4 & 1.52 & 0.34 & 0.23 & 0.56 & 0.57 & 0.78 & Stable \\
\hline & Case 5 & 2.16 & 2.97 & 0.42 & 0.41 & 0.45 & 0.12 & Stable \\
\hline \multirow{5}{*}{$\begin{array}{l}\text { Line to Line } \\
\text { (L-L) }\end{array}$} & Case 1 & 0.20 & 0.008 & 0.12 & - & - & - & Unstable \\
\hline & Case 2 & 0.20 & 0.0005 & 0.13 & - & - & - & Unstable \\
\hline & Case 3 & 0.24 & 0.0003 & 0.18 & - & - & - & Unstable \\
\hline & Case 4 & 0.48 & 0.0002 & 0.37 & 0.95 & 0.35 & 1.175 & stable \\
\hline & Case 5 & 0.64 & 0.0 & 0.98 & 0.71 & 0.0 & 0.45 & stable \\
\hline
\end{tabular}

Finally, it can be concluded from these case studies that selection of reactive control gain is crucial for STVS improvement in a highly three-phase PV penetrated DN following unsymmetrical faults. Furthermore, though most of the researches presented in the literature avoid peak current limit control, it has significant impact on the STVS while $k_{q}$ is other than 1.0.

\section{B. Case-studies on IEEE 13 bus Test-system:}

Typically, most of the residential networks are characteristically unbalanced due to the uneven loads in different phases, line configuration and unequal PV penetrations. Therefore, it is essential to conduct the case studies in unbalanced network to reveal the precise conclusions regarding the STVS for the purpose of assisting DN design engineers. Consequently, in this section, the case-studies are accomplished in IEEE 13 bus test-system which represents an unbalanced DN. The specifications of this test-system have been given in [18]. It needs to mention that the IEEE 13 bus test-system has been modified by connecting PV units at nodes $675(1.0 \mathrm{pu}), 671(0.5 \mathrm{pu})$ and $634(0.5 \mathrm{pu})$ as shown in Fig. 4, and adopting approximately 55\% IM-type load. The revised loads are accessible in Table A3 in Appendix A.

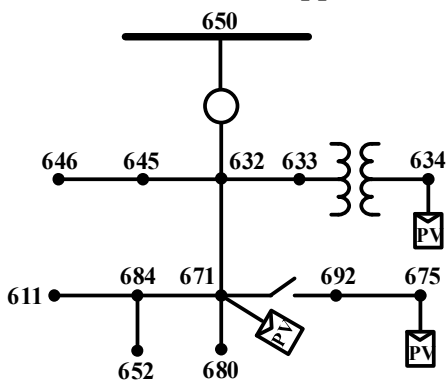

Figure 4. Revised IEEE 13 bus test-system.
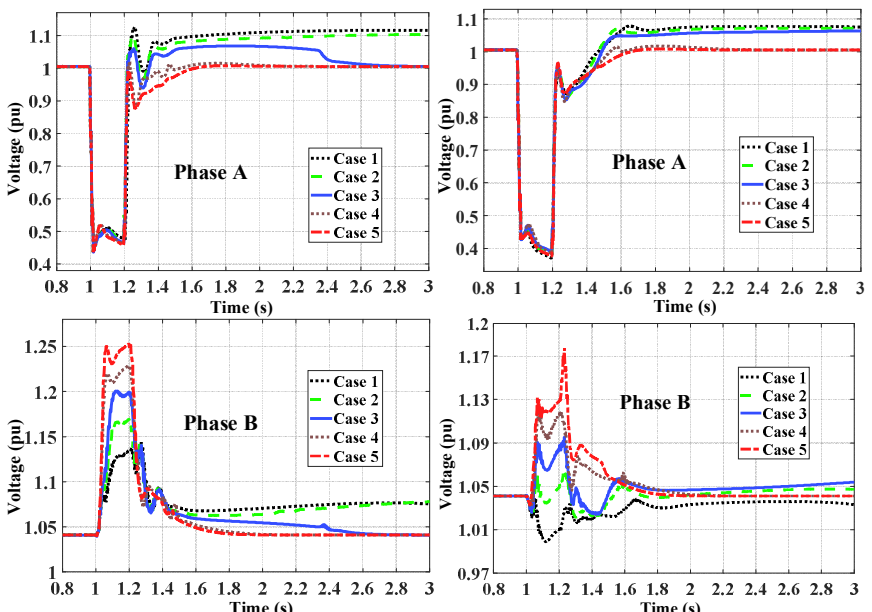

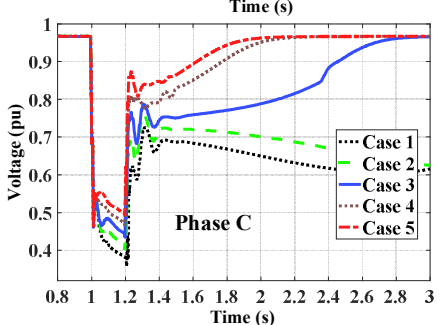

(a)

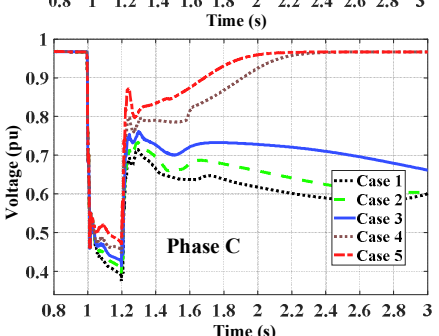

(b)
Figure 5. Voltage dynamics at node 675: (a) without and (b) with $I$ limit.

According to the structure of IEEE 13 bus, node 675 is the only remotely located three-phase node which is connected with large loads. Therefore, the voltage profile at node 675 is focused and measured without and with $I$ limit and presented in Fig. 5. Unlike IEEE 4 bus systems, it can be observed from Fig. 5(a) that STVI is occurred in Cases 1 and 2, whilst postcontingency voltage has been recovered in Cases 3 to 5 though slow-recovery can be seen in Case 3 . This is owing to low voltage profile in Phase $\mathrm{C}$ and unbalance nature of the network. On the other hand, Phase B has been experienced voltage swell nearly $0.225 \mathrm{pu}$ and $0.25 \mathrm{pu}$, respectively in 
Cases 4 and 5, which are higher than the international requirement [16, 17], and can cause of undesired inverter disconnection. It is clear that the system was able to limit the voltage swell within $0.2 \mathrm{pu}$ while avoiding instability only in Case 3. However, the scenario is different in case of $I$ limit control which is shown in Fig. 7 (b). As can be seen, STVI is occurred in Cases 1 to 3 . On the other hand, both maximum voltages swell limit within 0.2 pu in Phase B and recovery of Phase $\mathrm{A}$ and $\mathrm{C}$ voltages to stable condition have been confirmed in Cases 4 and 5. Therefore, the conclusions drawn in IEEE 4 bus system as selection of reactive control gain is crucial in STVS improvement have been re-confirmed in this case study as well. Additionally, it has been shown that STVI could be more severe in unbalanced network, for example Cases 1 and 2 without $I$ limit control.

\section{CONCLUSIONS}

This paper has intensively investigated the STVS of highly three-phase PV penetrated DNs following asymmetrical faults through injecting different-level of negative sequence power. Case studies are conducted on balanced IEEE 4 bus and unbalanced IEEE 13 bus systems. It is perceived that STVS of a balanced DN would be impaired while the penetration of negative sequence power is increased. Additionally, if the gridtied inverter applies peak current limit control, STVI can be happened. In contrast, STVI is likely to be occurred even without peak current limit control in unbalanced DN in case of high negative sequence power penetration. However, penetrating more positive sequence power can assist in avoiding possible voltage collapse, although the risk inverter disconnection due to high voltage swell would be present. Therefore, to ensure system security in terms of STVS and voltage swell, optimal injection of negative sequence power plays the key role that would need to be determined carefully based on the network characteristics. Finally, it is believed that this study would be useful for engineers in planning the PV installation in DNs considering the security.

\section{APPENDIX A}

TABLE A1: REVISED LOADS OF IEEE 4 BUS SYSTEM.

\begin{tabular}{|c|c|c|c|c|c|}
\hline \multirow{2}{*}{ Phase } & \multicolumn{2}{|c|}{$\mathrm{kW}$} & \multicolumn{2}{|c|}{ kVAr } & \multirow{2}{*}{$\mathrm{kVA}$} \\
\hline & $\mathrm{IM}$ & ZIP & IM & ZIP & \\
\hline $\mathrm{A}$ & 400 & 200 & 251 & 40 & 667 \\
\hline B & 400 & 200 & 251 & 40 & 667 \\
\hline $\mathrm{C}$ & 400 & 200 & 251 & 40 & 667 \\
\hline Total & 1200 & 600 & 753 & 120 & 2001 \\
\hline
\end{tabular}

TABLE A2: PU PARAMETERS OF IM LOAD [8, 19].

\begin{tabular}{|c|c|c|c|c|c|c|}
\hline $\mathrm{R}_{\mathrm{s}}$ & $\mathrm{X}_{\mathrm{s}}$ & $\mathrm{R}_{\mathrm{r}}$ & $\mathrm{X}_{\mathrm{r}}$ & $\mathrm{X}_{\mathrm{m}}$ & $\mathrm{H}$ & $\mathrm{p}$ \\
\hline 0.1 & 0.1 & 0.09 & 0.06 & 2.5 & .28 & 2 \\
\hline
\end{tabular}

TABLE A3: MODIFIED LOADS OF IEEE 13 NODE TEST FEEDER.

\begin{tabular}{|c|c|c|c|c|c|c|c|c|c|c|c|c|}
\hline \multirow{3}{*}{ Bus no } & \multicolumn{6}{|c|}{ ZIP load } & \multicolumn{6}{|c|}{ IM load } \\
\hline & \multicolumn{3}{|c|}{$\mathrm{kW}$} & \multicolumn{3}{|c|}{ kVAr } & \multicolumn{3}{|c|}{$\mathrm{kW}$} & \multicolumn{3}{|c|}{ kVAr } \\
\hline & $\mathrm{A}$ & B & $\mathrm{C}$ & A & B & $\mathrm{C}$ & $\mathrm{A}$ & B & $\mathrm{C}$ & $\mathrm{A}$ & B & $\mathrm{C}$ \\
\hline 675 & 185 & 13 & 52 & 0 & 25 & 62 & 300 & 55 & 238 & 189 & 35 & 150 \\
\hline 671 & 100 & 100 & 100 & 41 & 41 & 41 & 285 & 285 & 285 & 179 & 179 & 179 \\
\hline $\begin{array}{c}671 \\
\text { (DL) }\end{array}$ & 17 & 26 & 32 & 10 & 13 & 15 & 0 & 40 & 85 & 0 & 25 & 53 \\
\hline 634 & 41 & 18 & 18 & 35 & 26 & 26 & 119 & 102 & 102 & 75 & 64 & 64 \\
\hline 611 & & & 170 & & & 80 & & & & & & \\
\hline 652 & 128 & & & 86 & & & & & & & & \\
\hline
\end{tabular}

\begin{tabular}{|c|c|c|c|c|c|c|c|c|c|c|c|c|}
\hline 645 & & 170 & & & 125 & & & & & & & \\
\hline 646 & & 230 & & & 132 & & & & & & & \\
\hline 692 & & & 51 & & & 76 & & & 119 & & & 75 \\
\hline Total & 471 & 557 & 422 & 172 & 362 & 300 & 704 & 482 & 829 & 443 & 303 & 521 \\
\hline
\end{tabular}

\section{REFERENCE}

[1] "Snapshot of global photovoltaic markets 2017, Report IEA-PVPS T133," IEA-PVPS2018.

[2] J. L. Sosa, M. Castilla, J. Miret, J. Matas, and Y. A. Al-Turki, "Control Strategy to Maximize the Power Capability of PV Three-Phase Inverters During Voltage Sags," IEEE Transactions on Power Electronics, vol. 31, no. 4, pp. 3314-3323, 2016.

[3] A. Camacho, M. Castilla, J. Miret, A. Borrell, and L. G. de Vicuña, "Active and reactive power strategies with peak current limitation for distributed generation inverters during unbalanced grid faults," IEEE Transactions on industrial electronics, vol. 62, no. 3, pp. 1515-1525, 2015.

[4] K. Kawabe, Y. Ota, A. Yokoyama, and K. Tanaka, "Novel Dynamic Voltage Support Capability of Photovoltaic Systems for Improvement of Short-Term Voltage Stability in Power Systems," IEEE Transactions on Power Systems, vol. 32, no. 3, pp. 1796-1804, 2017.

[5] M. Islam, N. Mithulananthan, and M. J. Hossain, "Dynamic voltage support by TL-PV systems to mitigate short-term voltage instability in residential DN," IEEE Transactions on Power Systems, vol. PP, no. 99, pp. 1-1, 2017.

[6] K. Kawabe and K. Tanaka, "Impact of Dynamic Behavior of Photovoltaic Power Generation Systems on Short-Term Voltage Stability," Power Systems, IEEE Transactions on, vol. 30, no. 6, pp. 3416-3424, 2015.

[7] M. Islam, M. Nadarajah, and J. Hossain, "Dynamic behavior of transformerless PV system on the short-term voltage stability of distribution network," in Power \& Energy Society General Meeting, 2017 IEEE, 2017, pp. 1-5: IEEE.

[8] M. Islam, M. Nadarajah, and J. Hossain, "Short-term Voltage Stability Enhancement in Residential Grid with High Penetration of Rooftop PV units," IEEE Transactions on Sustainable Energy, 2018.

[9] Y. Ma, G. G. Karady, and L. Breazeale, "Short-term voltage stability analysis for power system with single-phase motor load," in 2012 IEEE Power and Energy Society General Meeting, 2012, pp. 1-6.

[10] K. Ma, W. Chen, M. Liserre, and F. Blaabjerg, "Power controllability of a three-phase converter with an unbalanced AC source," IEEE Transactions on Power Electronics, vol. 30, no. 3, pp. 1591-1604, 2015.

[11] J. L. Sosa, M. Castilla, J. Miret, J. Matas, and Y. Al-Turki, "Control strategy to maximize the power capability of PV three-phase inverters during voltage sags," IEEE Transactions on Power Electronics, vol. 31, no. 4, pp. 3314-3323, 2016.

[12] X. Guo, X. Zhang, B. Wang, W. Wu, and J. M. Guerrero, "Asymmetrical grid fault ride-through strategy of three-phase grid-connected inverter considering network impedance impact in low-voltage grid," IEEE Transactions on Power Electronics, vol. 29, no. 3, pp. 1064-1068, 2014

[13] F. Nejabatkhah, Y. W. Li, and B. Wu, "Control strategies of three-phase distributed generation inverters for grid unbalanced voltage compensation," IEEE Transactions on Power Electronics, vol. 31, no. 7, pp. 5228-5241, 2016.

[14] J. Jia, G. Yang, and A. H. Nielsen, "A Review on Grid-Connected Converter Control for Short-Circuit Power Provision Under Grid Unbalanced Faults," IEEE Transactions on Power Delivery, vol. 33, no. 2, pp. 649-661, 2018.

[15] A. Camacho, M. Castilla, J. Miret, J. C. Vasquez, and E. Alarcón-Gallo, "Flexible voltage support control for three-phase distributed generation inverters under grid fault," IEEE Transactions on Tndustrial Electronics, vol. 60, no. 4, pp. 1429-1441, 2013.

[16] M. B. Seon Gu Kim, STRI, "Towards the development of a set of grid code requirements for wind farms: Transient reactive power requirements (Part 3 Report of Vindforsk Project V-369)," 2013.

[17] M. Tsili and S. Papathanassiou, "A review of grid code technical requirements for wind farms," IET Renewable Power Generation, vol. 3 , no. 3, pp. 308-332, 2009.

[18] Power and Energy Society, 'Standard 4-bus Cases and 13-bus Feeder'. Available: http://ewh.ieee.org/soc/pes/dsacom/testfeeders/, Retived on 30.10.2016

[19] C. W. Taylor, Power system voltage stability. McGraw-Hill, 1994. 